\title{
Serial QuantiFERON TB-2G Testing Over a Four-year Period in Healthcare Workers at a City Hospital
}

\author{
Tomohiro Uto ${ }^{1,2}$, Kazumasa Yasuda ${ }^{1,2}$, Shinya Sagisaka ${ }^{1}$, Jun Sato ${ }^{1}$, Shiro Imokawa ${ }^{1}$, \\ Noriko Uemura ${ }^{2}$, Takafumi Suda ${ }^{3}$ and Kingo Chida ${ }^{3}$
}

\begin{abstract}
Objective To evaluate the performance and practicality of QuantiFERON TB-2G (QFT-2G) testing for screening healthcare workers (HCWs) at a city hospital in Japan without a tuberculosis (TB)-specific ward. Methods We performed a chart review of $951 \mathrm{HCWs}(251$ men and 700 women) who underwent QFT-2G testing as a part of their pre-employment or annual employee screening between April 2007 and March 2010. Results The initial QFT-2G test was interpreted as positive in $28(2.9 \%)$ HCWs, negative in 884 HCWs (92.9\%) and indeterminate in $39 \mathrm{HCWs}(4.1 \%)$. During the four-year study period, $37 \mathrm{HCWs}$ were diagnosed as being positive at least once. Nine $(0.98 \%)$ of the $923 \mathrm{HCWs}$ with indeterminate or negative results on the initial testing converted to a positive status, including 6/479 (1.25\%) nurses, $2 / 100(2.0 \%)$ office staff members and $1 / 147(0.68 \%)$ physicians. No HCWs with a positive result had a history of tuberculosis (TB) or any apparent contact with active TB patients and did not opt for treatment of latent TB. Seven (25\%) of the $28 \mathrm{HCWs}$ who were determined to be positive on the initial testing reverted to an indeterminate or negative status.
\end{abstract}

Conclusion In a series of annual serial QFT-2G tests, some HCWs exhibited conversion and/or reversion. Therefore, caution is required when interpreting mild fluctuations in interferon- $\gamma$ responses.

Key words: interferon-gamma release assay, tuberculosis, infection control, healthcare workers

(Intern Med 53: 1119-1124, 2014)

(DOI: 10.2169/internalmedicine.53.1288)

\section{Introduction}

Healthcare workers (HCWs) are at increased risk of infection due to occupational exposure to tuberculosis (TB) patients. TB patients are incidentally referred to the hospital before a definitive diagnosis is confirmed, even if the hospital does not have a TB-specific ward. Yamauchi et al. reported that female nurses have a two-fold higher risk of developing active TB than the general population in Japan (1). Therefore, preventing active TB is a fundamental challenge with respect to infection control in hospitals.

Two important components of TB infection control are routine screening for latent TB infection (LTBI) and the administration of chemoprophylaxis among HCWs. Based on the recommendation for the sequential performance of the two-step tuberculin skin test (TST) and the standard TST for newly hired HCWs (2), prior to 2006, we performed the TST in our hospital as an effective strategy for preventing nosocomial TB. However, the accuracy of the diagnosis of LTBI obtained with TST is sometimes doubtful due to the possibility of false-positive results in individuals who have received Bacillus Calmette-Guérin (BCG) vaccination and/or been exposed to non-tuberculosis mycobacterium infection (3).

A new diagnostic test for LTBI, the interferon (IFN)- $\gamma$ release assay (IGRA), has recently been developed to screen individuals with a history of TB contact, immigrants and other high-risk groups (4-6). The IGRA has a higher specificity than TST, avoids the use of subjective measurements, can be repeated without boosting and eliminates the need for repeat visits and two-step testing. Vinton et al. suggested

${ }^{1}$ Department of Respiratory Medicine, Iwata City Hospital, Japan, ${ }^{2}$ Infection Control Team, Iwata City Hospital, Japan and ${ }^{3}$ The Second Division, Department of Internal Medicine, Hamamatsu University School of Medicine, Japan

Received for publication July 2, 2013; Accepted for publication December 26, 2013

Correspondence to Dr. Tomohiro Uto, t-uto@hospital.iwata.shizuoka.jp 
Table 1. Characteristics of Participants who Underwent QFT-2G Testing

\begin{tabular}{lc}
\hline Characteristic & $\mathrm{n}=951$ \\
\hline Sex $(\mathrm{M} / \mathrm{F})$ & $251 / 700$ \\
Age, median (range), years & $32(19-67)$ \\
$<29(\%)$ & $274(28.8)$ \\
$30-39$ & $346(36.4)$ \\
$40-49$ & $183(19.2)$ \\
$50-59$ & $124(13.0)$ \\
$>60$ & $24(2.5)$ \\
Occupation & \\
Nurse (\%) & $492(51.7)$ \\
Physician, dentist & $151(15.9)$ \\
Office staff member & $106(11.1)$ \\
Medical technician & $89(9.6)$ \\
Midwife & $35(3.7)$ \\
Physiotherapist & $28(2.9)$ \\
Pharmacologist & $20(2.1)$ \\
Nursery teacher & $15(1.6)$ \\
Dietitian & $10(1.1)$ \\
Dental corpsman & $5(0.5)$ \\
\hline
\end{tabular}

that the QuantiFERON-TB (QFT) test is more effective for identifying HCWs with LTBI in countries with a low prevalence of TB due to the elimination of the possibility of false-positive results caused by BCG vaccination (7). Currently, most healthcare sector employees in North America undergo testing at the time of recruitment (8). In Japan, there has been a steady increase in the number of medical facilities that request QFT test results as part of their preemployment examination. The main purpose of preemployment QFT testing is to identify the baseline immune status of each HCW, as LTBI can be screened if HCWs have been in contact with TB patients.

Since 2007, the QFT-2G test has been performed as a pre-employment and annual medical examination in our hospital. Few previous reports have examined serial QFT-2G tests over a four-year period. The present study aimed to evaluate the efficacy and practicality of QFT-2G testing for four years in HCWs at a second-level healthcare hospital without a TB-specific ward.

\section{Materials and Methods}

\section{Participants}

We examined all QFT records of HCWs at Iwata City Hospital (Shizuoka, Japan) between April 2007 and March 2010. This institution is a 500-bed second-level healthcare hospital without a TB-specific ward. In this hospital, the ratio of the number of cases of smear-positive TB/number of outpatients per year was $25 / 39,337$ in $2006,29 / 38,814$ in 2007, 27/35,941 in 2008, 10/35,214 in 2009 and 28/32,580 in 2010. All HCWs at our institution received QFT-2G testing for pre-employment or as an annual medical examination. The HCWs included physicians, dentists, nurses, midwives, physiotherapists, dental corpsmen, pharmacologists, medical technicians, office staff members, nursery teachers
Table 2. Number of HCWs who Received Annual QFT Testing from 2007

\begin{tabular}{lcc}
\hline Year & $\begin{array}{c}\text { HCWs received annual } \\
\text { QFT test from 2007 }\end{array}$ & $\begin{array}{c}\text { HCWs received QFT test in } \\
\text { each year }\end{array}$ \\
\hline 2007 & 518 & 518 \\
2008 & 490 & 578 \\
2009 & 433 & 721 \\
2010 & 382 & 851 \\
\hline In each year, the numbers of new employees and HCWs without \\
QFT-2G in prior years were included.
\end{tabular}

and dietitians. Information regarding the following variables was collected using a questionnaire: history of prior TB or exposure to TB patients; the presence of underlying diseases, such as malignant disease or diabetes mellitus; and the use of medications, such as corticosteroids or immunosuppressants.

A total of $951 \mathrm{HCWs}$ (251 men and 700 women; median age, 32 years; range 19-67 years) underwent QFT-2G testing (Table 1). The subjects were divided into five groups according to age, as follows: $274(28.8 \%)$ subjects were $\leq 29$ years, $346(36.4 \%)$ subjects were 30-39 years, $183(19.2 \%)$ subjects were 40-49 years, $124(13.0 \%)$ subjects were 50-59 years and $24(2.5 \%)$ subjects were $\geq 60$ years.

Of the 518 HCWs who underwent QFT-2G testing in 2007, 382 underwent annual QFT testing for four consecutive years (Table 2). The number of HCWs who underwent QFT testing each year was 518 in 2007, 578 in 2008, 721 in 2009 and 851 in 2010.

\section{QFT-2G testing}

The QFT-2G tests were performed according to the manufacturer's recommendations (Cellestis, Carnegie, Australia). The test results were considered positive if the concentration of IFN- $\gamma$ in the sample well following stimulation with the 6-kDa early secretory antigenic target of Mycobacterium tuberculosis (ESAT-6) and/or culture filtrate protein (CFP)-10 was $\geq 0.35 \mathrm{IU} / \mathrm{mL}$. The results were considered indeterminate if the IFN- $\gamma$ level was $\geq 0.10 \mathrm{IU} / \mathrm{mL}$ and $<0.35 \mathrm{IU} / \mathrm{mL}$ in both antigen wells. The results were considered negative if the IFN- $\gamma$ level was $<0.10 \mathrm{IU} / \mathrm{mL}$ in both antigen wells.

We interpreted QFT conversion using the definition recommended by the US Centers for Disease Control and Prevention (CDC) (9), i.e., a baseline IFN- $\gamma$ level of $<0.35$ IU/ $\mathrm{mL}$ and a follow-up IFN- $\gamma$ level of $\geq 0.35 \mathrm{IU} / \mathrm{mL}$. QFT reversion was defined as a baseline IFN- $\gamma$ level of $\geq 0.35$ IU/ $\mathrm{mL}$ with a follow-up IFN- $\gamma$ level of $<0.35 \mathrm{IU} / \mathrm{mL}$.

QFT-2G testing was performed over a period of four years. We evaluated the performance and practicality of this modality for screening HCWs at our hospital.

\section{Statistical analysis}

All values were analyzed using the Dr. SPSS II for Windows software program (SPSS Inc., Chicago, USA). For the statistical analyses of the results of the initial QFT-2G testing and the characteristics of the positive, indeterminate and 
Table 3. Results of Initial QFT-2G Testing and Characteristics of Each Group

\begin{tabular}{lccc}
\hline & Positive & Indeterminate & Negative \\
\hline Number $(\%)$ & $28(2.9)$ & $39(4.1)$ & $884(92.9)$ \\
Age, median (range), years & $44(22-64) *$ & $31(22-59)$ & $32(19-66)$ \\
Sex $(\mathrm{M} / \mathrm{F})$ & $10 / 18$ & $9 / 30$ & $232 / 652$ \\
ESAT- $6^{\dagger}$, IFN- $\gamma(\mathrm{IU} / \mathrm{mL})$ & $0.40 \pm 0.61$ & & \\
$\mathrm{CFP}-10^{\dagger}, \mathrm{IFN}-\gamma(\mathrm{IU} / \mathrm{mL})$ & $1.03 \pm 1.23$ & \\
\hline *Significant difference from negative group $(\mathrm{p}<0.05)$ & \\
${ }^{\dagger}$ Data are presented as mean \pm standard deviation.
\end{tabular}

Table 4. Incidence of Conversion in QFT-2G Negative HCWs

\begin{tabular}{|c|c|c|c|c|}
\hline & $\begin{array}{c}\text { Number } \\
\text { of HCWs } \\
\text { with } \\
\text { negative } \\
\text { at initial } \\
\text { test }\end{array}$ & $\begin{array}{c}\text { Number } \\
\text { of HCWs } \\
\text { with } \\
\text { QFT-2G } \\
\text { conversion }\end{array}$ & $\begin{array}{c}\% \\
\text { converting }\end{array}$ & $\begin{array}{c}\text { OR } \\
(95 \% \mathrm{CI})\end{array}$ \\
\hline \multicolumn{5}{|l|}{ Sex } \\
\hline Male & 242 & 0 & 0 & \\
\hline Female & 681 & 9 & 1.32 & \\
\hline \multicolumn{5}{|l|}{ Age (years) } \\
\hline$<29$ & 269 & 1 & 0.37 & Reference \\
\hline $30-39$ & 341 & 3 & 0.88 & $2.38(0.28-34.23)$ \\
\hline $40-49$ & 176 & 2 & 1.14 & $3.08(0.42-51.47)$ \\
\hline $50-59$ & 118 & 2 & 1.69 & $4.62(0.41-51.47)$ \\
\hline$>60$ & 19 & 1 & 5.26 & $14.89(0.89-247.96)$ \\
\hline \multicolumn{5}{|l|}{ Occupation } \\
\hline Nurse & 479 & 6 & 1.25 & Reference \\
\hline Physician, dentist & 147 & 1 & 0.68 & $0.54(0.06-4.52)$ \\
\hline Office staff member & 100 & 2 & 2.00 & $1.61(0.32-8.09)$ \\
\hline Medical technician & 86 & 0 & 0 & \\
\hline Midwife & 34 & 0 & 0 & \\
\hline Physiotherapist & 28 & 0 & 0 & \\
\hline Pharmacologist & 19 & 0 & 0 & \\
\hline Nursery teacher & 15 & 0 & 0 & \\
\hline Dietitian & 10 & 0 & 0 & \\
\hline Dental corpsman & 5 & 0 & 0 & \\
\hline
\end{tabular}

negative groups, the Mann-Whitney $U$ test was used. A value of $\mathrm{p}<0.05$ was considered to indicate statistical significance. The odds ratio (OR) and confidence interval (CI) were calculated for the single-variable analysis of the rate of conversion.

\section{Results}

\section{Initial QFT-2G testing of the HCWs}

Regarding the initial QFT-2G tests, 28 (2.9\%) of 951 tests were interpreted as positive, $884(92.9 \%)$ were interpreted as negative and $39(4.1 \%)$ were interpreted as indeterminate. The median age of the HCWs with a positive result was 44 years (range, 22-64 years), which was significantly higher than that of the HCWs with a negative result $(\mathrm{p}<0.05$, Table 3). The mean IFN- $\gamma$ response level for ESAT-6 was $0.40 \pm 0.61 \mathrm{IU} / \mathrm{mL}$, while that for CFP-10 was $1.03 \pm 1.23 \mathrm{IU} /$ $\mathrm{mL}$ among the HCWs with a positive result. The rate of positive results in each year was $2.9 \%$ in $2007,3.8 \%$ in

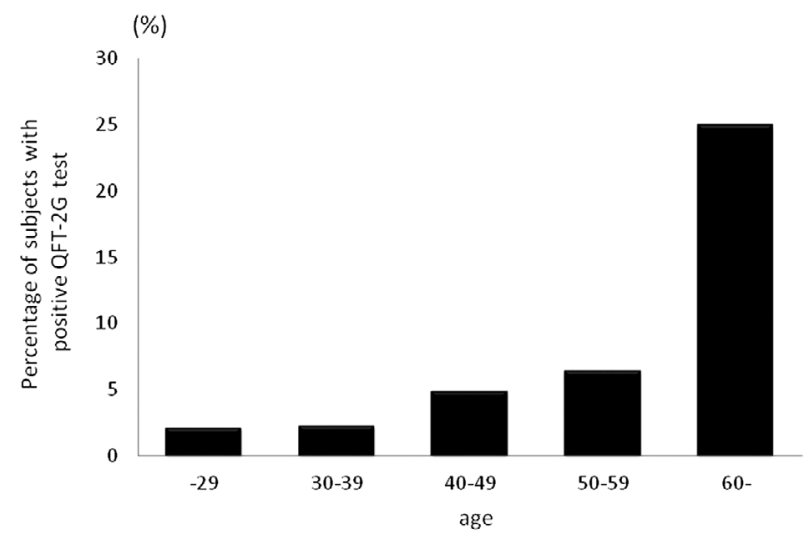

Figure 1. Percentage of subjects with at least one positive QFT-2G test result during the four-year observation period according to age. The rate of positive results increased with age.

2008, 2.9\% in 2009 and $3.1 \%$ in 2010 , with no significant differences between these values.

\section{Chronological observation of the HCWs with a posi- tive QFT-2G test result}

During the four-year observation period, $37 \mathrm{HCWs}$ (nine men and 28 women) had at least one positive QFT-2G test result. Chest computed tomography did not show any abnormal findings associated with mycobacterial infection, such as M. tuberculosis or M. kansasii.

The median age of these subjects was 47 years (range, 24-66 years), including $19 / 492(3.86 \%)$ nurses, 8/106 $(7.55 \%)$ office staff members, 5/151 $(3.31 \%)$ physicians, 3/ $89(3.37 \%)$ medical technicians, $1 / 20(5.00 \%)$ pharmacologists and $1 / 35(2.85 \%)$ midwives. According to age, the rate of positive results increased from $2.2 \%$ among the subjects $\leq 29$ years of age to $25 \%$ among those $\geq 60$ years of age (Fig. 1).

A total of $923 \mathrm{HCWs}$ had results that were interpreted as negative or indeterminate on the initial testing, nine $(0.98 \%)$ of whom converted to positive results. All of these subjects were women, including 6/479 (1.25\%) nurses, 2/100 $(2.00 \%)$ office staff members and $1 / 147(0.68 \%)$ physicians (Table 4). Five and six subjects converted with ESAT-6 and CFP-10, respectively, while two subjects converted with both (Fig. 2A, B). None of these subjects had a history of TB or exposure to active TB patients, and none opted for treatment of LTBI.

Seven of the $28 \mathrm{HCWs}(25.0 \%)$ with a positive result on the initial testing reverted to an indeterminate or negative status over the four-year observation period. Four of these seven HCWs were women, and there were no significant differences in the rate of reversion between the generations (Table 5). This group consisted of $2 / 13$ (15\%) nurses, 3/6 $(50 \%)$ office staff members, $1 / 3(33 \%)$ medical technicians and one $(100 \%)$ pharmacologist. Three and six subjects reverted with ESAT-6 and CFP-10, respectively, while two reverted with both (Fig. 2C, D). 


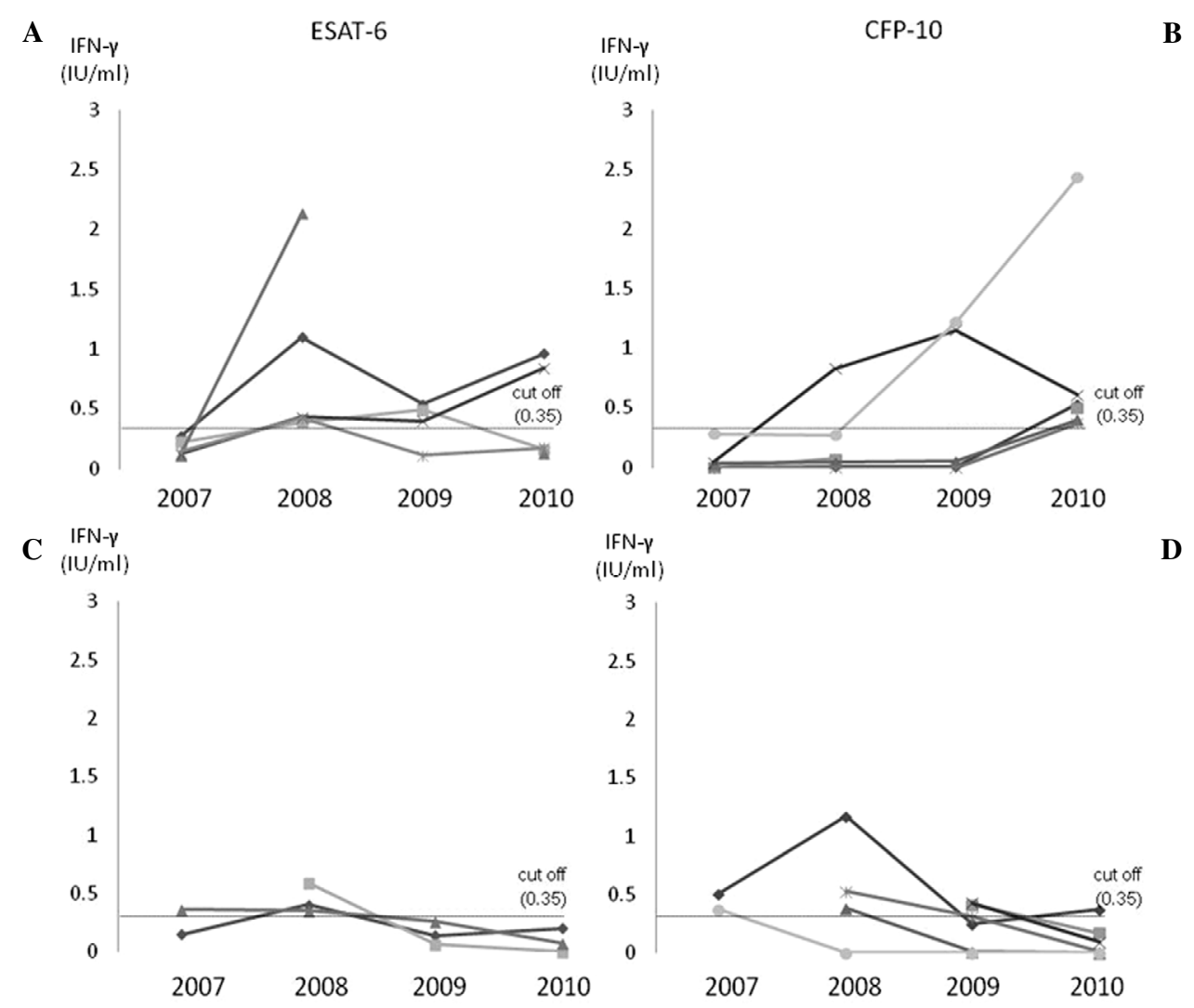

Figure 2. The ESAT-6 and CFP-10 levels in the subjects who converted to positive QFT-2G test results. For ESAT-6, five subjects converted to positive results, two of whom reverted to negative results (A). For CFP-10, all subjects who converted to positive test results remained positive (B). The ESAT-6 and CFP-10 levels in the subjects who reverted to negative QFT-2G test results. For ESAT-6, no subjects converted again after exhibiting reversion to negative results (C). For CFP-10, only one subject reverted to a positive test result (D).

Table 5. Incidence of Reversion in QFT-2G Positive HCWs

\begin{tabular}{|c|c|c|c|c|}
\hline & $\begin{array}{c}\text { Number } \\
\text { of HCWs } \\
\text { with } \\
\text { positive } \\
\text { at initial } \\
\text { test }\end{array}$ & $\begin{array}{c}\text { Number } \\
\text { of HCWs } \\
\text { QFT-2G } \\
\text { reversion }\end{array}$ & $\begin{array}{c}\% \\
\text { reverting }\end{array}$ & OR $(95 \% \mathrm{CI})$ \\
\hline \multicolumn{5}{|l|}{ Sex } \\
\hline Male & 9 & 3 & 33 & Reference \\
\hline Female & 19 & 4 & 21 & $0.53(0.04-3.14)$ \\
\hline \multicolumn{5}{|l|}{ Age (years) } \\
\hline$<29$ & 5 & 1 & 20 & Reference \\
\hline $30-39$ & 5 & 3 & 60 & $6.00(0.35-101.57)$ \\
\hline $40-49$ & 7 & 1 & 14 & $0.67(0.03-14.03)$ \\
\hline $50-59$ & 6 & 1 & 17 & $0.80(0.04-17.20)$ \\
\hline$>60$ & 5 & 1 & 20 & $1.00(0.05-22.18)$ \\
\hline \multicolumn{5}{|l|}{ Occupation } \\
\hline Nurse & 13 & 2 & 15 & Reference \\
\hline Physician, dentist & 4 & 0 & 0 & \\
\hline Office staff member & 6 & 3 & 50 & $5.50(0.61-49.54)$ \\
\hline Medical technician & 3 & 1 & 33 & $2.75(0.16-46.79)$ \\
\hline Midwife & 1 & 0 & 0 & \\
\hline Physiotherapist & 0 & 0 & 0 & \\
\hline Pharmacologist & 1 & 1 & 100 & \\
\hline Nursery teacher & 0 & 0 & 0 & \\
\hline Dietitian & 0 & 0 & 0 & \\
\hline Dental corpsman & 0 & 0 & 0 & \\
\hline
\end{tabular}

\section{Discussion}

In this study, serial QFT-2G testing was performed at the pre-employment stage or during annual medical examinations between 2007 and 2010 among HCWs at a city hospital without a TB-specific ward. Each year, the rate of positive QFT-2G testing was approximately 3\%. Although no HCWs with positive results had a medical history of TB or apparent contact with active TB patients, $0.98 \%$ of the HCWs with negative or indeterminate initial test results converted to positive results.

In Japan, 23,000 incident cases of TB were reported in 2010. Among them, $51 \%$ included elderly individuals $>70$ years of age, thus few incident cases of TB were reported among young people (10). HCWs are known to be at risk for TB $(11,12)$. Most HCWs are young and frequently have contact with elderly persons in routine practice. In order to prevent the spread of TB, infection control policies in many hospitals recommend the use of pre- and post-employment routine screening of HCWs using TST $(3,4)$. However, the usefulness of TST is limited by its lack of specificity. In contrast, the IGRA test is more specific because it uses antigens not shared by any of the BCG vaccine strains or the 
more common species of non-tuberculosis mycobacterium (13). The IGRA also has the advantage of exhibiting greater correlations with surrogate parameters of exposure to M. tuberculosis (14-16) and has a higher predictive value for LTBI progression to active TB $(17,18)$.

However, some individuals show positive results on QFT testing without a medical history of TB or exposure to active TB patients. With regard to the rate of positive QFT testing, Hotta et al. reported a proportion of $1.4 \%$ among 207 healthcare students in a population deemed to be at a low risk for TB in Japan (19). Among HCWs at a hospital with a TB-specific ward, Nakajima et al. showed a rate of positive QFT of approximately $10 \%$ (20). We adopted the QFT-2G for routine screening of HCWs at our hospital in 2007, and the rate of positive testing has been approximately $3 \%$ each year. There may be a relationship between the rate of positive QFT and the frequency of contact with hospitalized active TB patients. In the present study, the positive group was older than the negative group, which is in agreement with the findings of previous reports showing that the rate of positive QFT is higher among elderly persons (21-23). Furthermore, none of the HCWs in the positive group had a medical history of $\mathrm{TB}$ or exposure to active TB patients. QFT testing has been reported to have a high sensitivity and specificity for discriminating TB infection among low-risk groups (18); however, the positive predictive value in populations with a low prevalence of $\mathrm{TB}$ infection is significantly lower than that observed in populations with a high prevalence of TB. The QFT test may therefore not be effective when performed in populations with a low prevalence of $\mathrm{TB}$ infection due to the low positive predictive value.

In the present study, $0.98 \%$ (9/923) of the HCWs converted to positive results without developing TB, while $25 \%$ (7/28) reverted to negative results during the four-year observation period. With regard to occupation, there were no differences in the frequency of contact with TB patients between the groups, although we assumed that HCWs in close contact with TB patients exhibit a high conversion rate. Therefore, we may not have been able to detect the frequency of contact with patients as a risk factor for $\mathrm{TB}$ in our hospital because fewer TB patients visit our hospital compared with TB-specific hospitals.

In addition, among our HCWs who converted to positive results, the mean QFT-2G value was $0.35 \mathrm{IU} / \mathrm{mL}$, which indicates a cut-off value between a positive and indeterminate status. There are currently no data regarding the degree of the increase in the IFN- $\gamma$ response observed in patients with new TB infection as opposed to that due to test-related error or biological variation. Therefore, when interpreting test results according to current thresholds, the QFT is associated with a higher rate of conversion than the TST, with a 10mm increment threshold, raising the possibility that the IGRA is more sensitive in detecting recent conversion $(24,25)$. Pai et al. reported that it is uncertain whether a relatively small increase in the IFN- $\gamma$ response constitutes a true conversion. Therefore, the optimal threshold for distinguishing new infection from nonspecific variation must be determined (8). These authors also stated that it is important to consider the possibility that the sensitivity of IGRA for existing LTBI may be different from that for recent infection. The IGRA test may therefore facilitate the development of novel approaches to achieving nosocomial TB control. However, there is a potential risk of overtreatment, including the administration of LTBI therapy in uninfected HCWs, if nonspecific increases in IFN- $\gamma$ are misinterpreted as being serious. Several definitions for QFT conversion, other than that recommended by the $\mathrm{CDC}$, have been suggested, as follows: (1) a baseline IFN- $\gamma$ level of $<0.35 \mathrm{IU} / \mathrm{mL}$ with a follow-up IFN- $\gamma$ level of $\geq 0.35 \mathrm{IU} / \mathrm{mL}$, as well as a $30 \%$ increase in the IFN- $\gamma$ level over the baseline value (26); (2) a baseline IFN- $\gamma$ level of $<0.35 \mathrm{IU} / \mathrm{mL}$ with a follow-up IFN- $\gamma$ level of $\geq 0.35 \mathrm{IU} / \mathrm{mL}$, as well as an absolute increase in the IFN- $\gamma$ level of $0.35 \mathrm{IU} / \mathrm{mL}$ over the baseline value (8); and (3) a baseline IFN- $\gamma$ level of $<0.35 \mathrm{IU} / \mathrm{mL}$ with a follow-up IFN- $\gamma$ level of $\geq 0.70 \mathrm{IU} / \mathrm{mL}$ (8). However, which definition is the most appropriate is unclear. Well-controlled studies are required to further define the causes of individual variation in the IFN- $\gamma$ response, and physicians should provide careful observation taking into consideration the risk of TB infection in individuals in whom the value of QFT increases remarkably. However, interpreting QFT test results alone may result in misdiagnosis, especially in facilities with less contact with TB patients, as observed in our hospital.

Performing QFT testing is relatively expensive. However, Fox et al. reported that QFT screening reduces direct costs by reducing the number of diagnosed cases of LTBI, thus reducing the cost of treatment (27). In addition, in that study, QFT screening reduced the total number of clinic visits and the cohort exhibited increased adherence to TB prophylaxis compared with the TST cohort. The use of QFT results in superior clinical outcomes with lower costs than TST and should be considered in screening both non-BCG vaccinated and BCG-vaccinated new HCWs for LTBI (28). However, Ito reported that the estimated cost of annual QFT tests to prevent TB among female general nurses in Japan is high and therefore did not recommend the annual use of QFT tests (29).

Developing a uniform system of examination is difficult, as the frequency of contact with smear-positive patients differs among medical facilities. The frequency of QFT testing should be determined according to the frequency of contact with smear-positive patients. For example, high-risk groups should receive QFT testing every year, while low-risk groups should receive testing the at pre-employment examination or as needed. Annual QFT testing results in the easy diagnosis of LTBI because such testing demonstrates abnormal values different from conventional fluctuation in HCWs infected with TB. However, the need for annual QFT testing should be considered based on its cost-effectiveness. It is controversial whether QFT tests should be performed annually; therefore, the testing schedule must be determined at 
each medical facility.

Our study is associated with some limitations. First, QFT$2 \mathrm{G}$ results are affected by the time between sample collection and analysis as well as the method of preservation (30). We performed QFT-2G testing in our hospital laboratory. However, technical errors were unlikely to have affected our QFT-2G test results because we tested $>500$ subjects every year. Second, none of the HCWs with positive QFT-2G results opted for treatment of LTBI, although we recommended such therapy. Therefore, we were unable to investigate the course of QFT-2G testing after treatment of LTBI.

QFT-2G testing may facilitate the development of novel approaches for achieving nosocomial TB control among HCWs; however, some concerns remain. In the present series of annual QFT-2G tests, several patients exhibited conversion and/or reversion. Therefore, caution is required when interpreting mild fluctuations in the IFN- $\gamma$ response.

The authors state that they have no Conflict of Interest (COI).

\section{References}

1. Yamauchi Y. Tuberculosis risk of female nurses based on the Tuberculosis Surveillance Data. Kekkaku 74: 819-821, 1999 (in Japanese).

2. Moran-Mendoza O, Marion SA, Elwood K, et al. Tuberculin skin test size and risk of tuberculosis development: a large populationbased study in contacts. Int J Tuberc Lung Dis 11: 1014-1020, 2007.

3. Menzies D. What does tuberculin reactivity after Bacille CalmetteGuerin vaccination tell us? Clin Infect Dis 31 (Suppl 3): S71-S74, 2000.

4. Pai M, Riley LW, Colford JM Jr. Interferon- $\gamma$ assays in the immunodiagnosis of tuberculosis: a systematic review. Lancet Infect Dis 4: 761-776, 2004.

5. Dheda K, Udwadia ZF, Huggett JF, et al. Utility of the antigenspecific interferon- $\gamma$ assay for the management of tuberculosis. Curr Opin Pulm Med 11: 195-202, 2005.

6. CDC. Guidelines for preventing the transmission of Mycobacterium tuberculosis in health-care settings. MMWR 54: 1-141, 2005.

7. Vinton P, Mihrshahi S, Johnson P, et al. Comparison of QuantiFERON-TB Gold In-Tube Test and tuberculin skin test for identification of latent Mycobacterium tuberculosis infection in healthcare staff and association between positive test results and known risk factors for infection. Infect Control Hosp Epidemiol 30: 215-221, 2009.

8. Pai M, Joshi R, Dogra S, et al. Serial Testing of Health Care Workers for Tuberculosis Using Interferon- $\gamma$ Assay. Am J Respir Crit Care Med 174: 349-355, 2006.

9. Targeted tuberculin testing and treatment of latent tuberculosis infection. Am J Respir Crit Care Med 161: S221-S247, 2000.

10. Ministry of Health, Labour and Welfare in Japan. Number of registered patients with active TB and the rate of prevalence in 2010. Available from: http://www.mhlw.go.jp/bunya/kenkou/kekkakukansenshou03/dl/10.html

11. Menzies D, Joshi R, Pai M. Risk of tuberculosis infection and disease associated with work in health care settings. Int $\mathbf{J}$ Tuberc Lung Dis 11: 593-605, 2007.

12. Seidler A, Nienhaus A, Diel R. Review of epidemiological studies on the occupational risk of tuberculosis in low-incidence areas. Respiration 72: 431-446, 2005.

13. Nahid P, Pai M, Hopewell PC. Advances in the diagnosis and treatment of tuberculosis. Proc Am Thorac Soc 3: 103-110, 2006.

14. Diel R, Loddenkemper $R$, Meywald-Walter $K$, Gottschalk R, Nienhaus A. Comparative performance of tuberculin skin test, QuantiFERON-TB-Gold In Tube Assay, and T-Spot. TB test in contact investigations for tuberculosis. Chest 135: 1010-1018, 2009.

15. Diel R, Loddenkemper R, Nienhaus A. Evidence-based comparison of commercial interferon- $\gamma$ release assays for detecting active TB: a meta-analysis. Chest 137: 952-968, 2010.

16. Diel R, Loddenkemper R, Meywald-Walter K, Niemann S, Nienhaus A. Predictive value of a whole blood IFN- $\alpha$ assay for the development of active tuberculosis disease after recent infection with Mycobacterium tuberculosis. Am J Respir Crit Care Med 177: 1164-1170, 2008.

17. Diel R, Loddenkemper R, Niemann S, Meywald-Walter K, Nienhaus A. Negative and positive predictive value of a whole-blood Interferon- $\gamma$ release assay for developing active tuberculosis: an update. Am J Respir Crit Care Med 183: 88-95, 2011.

18. Mori T, Sakatani M, Yamagishi F, et al. Specific detection of tuberculosis infection: an interferon- $\gamma$-based assay using new antigens. Am J Respir Crit Care Med 170: 59-64, 2004.

19. Hotta K, Ogura T, Nishii K, et al. Whole blood Interferon-gamma assay for baseline tuberculosis screening among Japanese healthcare students. PLoS One 2: e803, 2007.

20. Nakajima Y, Ogata $H$, Yoshiyama $T$, et al. Results of repeated QFT-2G checkings at two-year interval and their comparison with TST results introduced into infection control program for staffs working at a hospital with isolation wards for tuberculosis. Kekkaku 83: 445-450, 2008 (in Japanese).

21. Harada N, Nakajima Y, Higuchi K, Sekiya Y, Rothel J, Mori T. Screening for tuberculosis infection using whole-blood interferon- $\gamma$ and Mantoux testing among Japanese healthcare workers. Infect Control Hosp Epidemiol 27: 442-448, 2006.

22. Mori T, Harada N, Higuchi K, Sekiya Y, Uchimura K, Shimao T. Waning of the specific interferon-gamma response after years of tuberculosis infection. Int J Tuberc Lung Dis 11: 1021-1025, 2007.

23. Suzuki K, Onozaki I, Shimura A, et al. QuantiFERON-TB-2 $2^{\text {nd }}$ Generation in the elderly person. Kekkaku 79: 200, 2004 (in Japanese).

24. Leyten EM, Mulder B, Prins C, et al. Use of enzyme-linked immunospot assay with Mycobacterium tuberculosis-specific peptides for diagnosis of recent infection with M. tuberculosis after accidental laboratory exposure. J Clin Microbiol 44: 1197-1201, 2006.

25. Anderson ST, Williams AJ, Brown JR, et al. Transmission of $M y$ cobacterium tuberculosis undetected by tuberculin skin testing. Am J Respir Crit Care Med 173: 1038-1042, 2006.

26. Veerapathran A, Joshi R, Goswami K, et al. T-cell assays for tuberculosis infection: deriving cut-offs for conversions using reproducibility data. PLoS ONE 3: e1850, 2008.

27. Fox BD, Kramer MR, Mor Z, et al. The QuantiFERON-TBGOLD assay for tuberculosis screening in healthcare workers: a cost-comparison analysis. Lung 187: 413-419, 2009.

28. de Perio MA, Tsevat J, Roselle GA, Kralovic SM, Eckman MH. Cost-effectiveness of interferon gamma release assays vs tuberculin skin tests in health care workers. Arch Intern Med 169: 179$187,2009$.

29. Ito K. Efficiency of periodic QuantiFERON-TB GOLD test in hospital nurse. Kekkaku 84: 709-712, 2009 (in Japanese).

30. Harada N. Interpretation of QFT results in several contact investigations. Kekkaku 85: 595-599, 2010 (in Japanese).

(C) 2014 The Japanese Society of Internal Medicine http://www.naika.or.jp/imonline/index.html 